\title{
Breast cancer associated mutations reported among Malaysian Malay, Chinese and Indian patients
}

\begin{abstract}
Breast cancer among women has reached an alarming stage compared to 20 years ago. It was found that single nucleotide polymorphisms (SNPs) play an important role in the development of breast cancer. SNPs may suppress or activate the functional genes, which then leads to an increased survival of cells due to the suppression of tumour suppressor genes or increased activity in detrimental proteins, resulting in cancers. Recently, breast cancer research is given significant attention due to the increasing number of breast cancer patients in Malaysia each year. However, breast cancer researches in Malaysia are mainly focused on well known breast cancer genes such as BRCA1 and BRCA2. There is less emphasis on the other breast cancer contributing genes. This paper was aimed to review the research findings on mutations associated with breast cancer in Malaysian Malay, Malaysian Chinese and Malaysian Indian as it has never been cumulatively reported and to ascertain the common mutations found in these three major Malaysia ethnicity.
\end{abstract}

Keyword: Breast cancer; SNPs; Malaysian Malay; Malaysian Chinese; Malaysian Indian; Mutations 